\title{
Preliminary Exploration of the Initial Diagnostic Prediction Model of Moderate Coronavirus Disease 2019 (2019-nCoV) Based on Clinical Data
}

\author{
Ritian Zha*, Jingmin Gui, Jiancheng Hao, Yungui Zhou, Wensheng Jiang, Shangming Chen, \\ Jiajia Zhao, Ruiping Xuan, Zhendong Jiang, Xiaoqin Liu, Ping Wang, Lei Zhang \\ Department of Respiratory Diseases, The First People's Hospital of Wuhu, Wuhu, China \\ Email: zrtanhui@126.com
}

How to cite this paper: Zha, R.T., Gui, J.M., Hao, J.C., Zhou, Y.G., Jiang, W.S., Chen, S.M., Zhao, J.J., Xuan, R.P., Jiang, Z.D., Liu, X.Q., Wang, P. and Zhang, L. (2021) Preliminary Exploration of the Initial Diagnostic Prediction Model of Moderate Coronavirus Disease 2019 (2019-nCoV) Based on Clinical Data. Open Journal of Nursing, 11, 7-16.

https://doi.org/10.4236/ojn.2021.111002

Received: December 7, 2020

Accepted: January 11, 2021

Published: January 14, 2021

Copyright $\odot 2021$ by author(s) and Scientific Research Publishing Inc. This work is licensed under the Creative Commons Attribution International License (CC BY 4.0).

http://creativecommons.org/licenses/by/4.0/

\section{(c) (i) Open Access}

\begin{abstract}
Objective: To explore those differences and relationships of the initial diagnostic clinical data between confirmed cases of 2019-nCoV and suspected cases of COVID-19, and then to establish prediction models for predicting the probability of the first diagnosis of 2019-nCoV. Methods: A total of 81 suspected cases and 87 confirmed cases of moderate 2019-nCoV diagnosed initially in the isolation wards of the First People's Hospital of Wuhu and the People's Hospital of Wuwei and Wuhan Caidian Module Hospital with the help of our hospital doctors were gathered, and retrospectively analyzed. Results: The most common symptoms were fever (76.79\%) and cough (64.29\%) in the total of 168 cases. The median age was 45 (35 - 56) years old in the 87 confirmed cases of moderate 2019-nCoV, older than the median age 36 (29$50)$ in the 81 suspected cases. There were significant more in the former than in the latter in the incidence of myalgia, ground-glass opacity (GGO), invasions of lesion in the peripheral lobes, vascular thickening and bronchial wall thickening, interlobular septal thicking, and small pulmonary nodules. On the contrary, there were less in the former than in the latter in the total number of leukocytes and neutrophils in blood routine examination and the levels of procalcitonin (PCT). Two groups were statistically significantly different $(P<0.05)$. Multivariate logistic regression analysis showed that age, fever, myalgia, GGO, vascular thickening and bronchial wall thickening, invasions of lesion in the peripheral lobes were independent factors for identification of 2019-nCoV, and the total number of leukocytes, cough, pharyngalgia and headache were negatively related. The established mathematical equation for
\end{abstract}

*The first author. 
predicting model for predicting the probability of the first diagnosis of 2019-nCoV is: $P=\mathrm{e}^{x} /\left(1+\mathrm{e}^{x}\right), x=-6.226+(0.071 \times$ ages $)+(1.720 \times$ fever $)+$ $(2.858 \times$ myalgia $)+(2.131 \times \mathrm{GGO})+(3.000 \times$ vascular thickening and bron-chial wall thickening $)+(3.438 \times$ invasions of lesion in the peripheral lobes $)+(-0.304 \times$ the number of leukocytes $)+(-1.478 \times$ cough $)+(-1.830 \times$ pharyngalgia $)+(-2.413 \times$ headache $)$, where $e$ is a natural logarithm. The area under the ROC curve (AUC) of this model was calculated to be 0.945 (0.915 $0.976)$. The sensitivity is 0.920 and the specificity is 0.827 when the appropriate critical point is 0.360 . Conclusions: A mathematical equation prediction model for predicting the probability of the first diagnosis of 2019-nCoV can be established based on the initial diagnostic clinical data of moderate 2019-nCoV. The prediction model is a good assistant diagnostic method for its high accurateness.

\section{Keywords}

Clinical Data, 2019-nCoV, Prediction Model

\section{Introduction}

During this current public health emergency of international concern, screening and diagnosing patients quickly to aid containment is a priority and these limitations make RT-PCR unsuitable for use in the field. Consequently, new tools are in great demand. Unfortunately, the sensitivity of the RNA test in the real world is not satisfactory. Clinical sensitivity of PCR decreases with days post symptom onset, Serological assay sensitivity increases with days post symptom onset [1]. Scholars ultimately found that computed tomography (CT) was associated with a higher rate of diagnostic accuracy than a real-time quantitative polymerase chain reaction (qPCR)-based approach. Even so, it is important that clinicians utilize a combination of laboratory and radiological testing where possible in order to ensure that this virus is reliably and quickly detected such that it may be treated and patients may be isolated in a timely fashion, thereby effectively curbing the further progression of this pandemic [2].

The population is generally susceptible to the $2019-\mathrm{nCoV}$. In order to prevent missed diagnosis, the diagnosis and treatment guidelines of the National Health Commission [3] point out in particular that suspected cases of 2019-nCoV can be screened first based on clinical manifestations, and then confirmed based on the new coronavirus nucleic acid detection, but the early clinical characteristics of suspected cases and confirmed cases have no significant difference [4], the detection rate of new coronavirus nucleic acid is very low [5], it is easy to delay diagnosis and treatment, and it is urgent to continue to develop new clinical diagnosis study of pneumonia caused by coronavirus infection. This study analyzes the clinical manifestations of common 2019-nCoV, screens out common 2019-nCoV risk factors, and initially explores a risk prediction model based on 
clinical manifestations, which is expected to help the clinical diagnosis of 2019-nCoV.

\section{Materials and Methods}

\subsection{Information}

This study is a retrospective case-control study that included the patients with the 2019-nCoV who were admitted to Wuhu First People's Hospital, Wuwei People's Hospital and Wuhan Development Zone Sports Center Fangcang Hospital from January 25, 2020 to March 6, 2020. There were 81 general suspected cases and 87 confirmed cases. The diagnosis of 2019-nCoV is based on the "New Coronavirus Pneumonia Diagnosis and Treatment Plan (Trial Sixth Edition)" by the National Health Commission [2]. The data of the patients were derived from the medical history collection, the first laboratory examination and the chest CT examination when the first-line clinicians received the patients. Data entry was separately recorded by the two groups consisted of different members of the project team and finally common compared and verified. The chest CT imaging omics data was obtained by the joint reading of the project team's imaging and respiratory experts.

\subsection{Statistical Methods}

Using SPSS 19.0 statistical software, some patients' peripheral blood C-reactive protein (CRP) and procalcitonin (PCT) vacancies were assigned missing values for statistical analysis. All observations were tested for normal distribution. Except for the age variable of the confirmed group which conformed to the normal distribution, the other variables did not conform to the normal distribution. Therefore, all measurement data are represented by the median and the 25th and 75th percentile [M (P25, P75)], and the comparison between groups is represented by the Kruskal-Wallis rank sum test; all the count data are represented by frequency and percentage (\%), The comparison between groups was tested by $\mathrm{X}^{2}$ or Fisher's exact probability method. The Bivariate process is used to analyze the correlation of various variables in the clinical manifestations, and then the two-class logistic unconditional regression analysis is used for the observations with potential correlations to preliminary explore the general 2019-nCoV first diagnosis prediction mathematical model.

\section{Results}

\subsection{General Information and Clinical Symptoms}

Among all 168 patients, 93 were male (55.36\%), and the median age was 39 (31 54) years old. Fever (76.79\%) and cough (64.29\%) were the most common symptoms. Between the two groups, the difference in patient age was statistically significant $(P=0.013)$. Among them, the minimum age of the 81 suspected cases was 5 years, the maximum age was 86 years, and the median age was 36 (29 - 50) 
years, The minimum age of patients in the confirmed 87 cases group is 8 years old, the oldest age is 90 years old, and the median age is 45 (35 - 56) years; in the clinical manifestations, 18 (20.69\%) patients in the confirmed group have muscle soreness, which is higher than 4 in the suspected group. (4.94\%) cases, the difference was significant $(P=0.003)$; there were no significant differences in the number of cases of fever, cough, sore throat, anorexia, diarrhea, headache, dizziness, chest tightness, and chest pain in the remaining clinical manifestations between the two groups $(P>0.005)$. See Table 1 for details.

\subsection{Auxiliary Inspection}

The peripheral blood median white blood cell count and neutrophil count (WBC, NEUT, $\times 10^{9} / \mathrm{L}$ ) of all ordinary patients were within the normal range, and the 81 suspected groups were $7.00(4.79,8.55)$ and 4.85 respectively. (3.17, $6.49)$, higher than $5.40(4.20,6.73)$ and $3.40(2.43,4.49)$ in the 87 confirmed group, the difference was statistically significant $(P<0.001)$. At the first diagnosis, the median PCT $(\mathrm{ng} / \mathrm{ml})$ of the 81 suspected groups was $0.26(0.14,0.35)$, which was higher than the 87 cases of the confirmed group of $0.12(0.05,0.26)$, the difference was significant $(P=0.011)$. However, The difference of the median CRP was not significant $(P=0.058), 18.35(6.60,43.80)$ in the suspected group and $9.81(4.43,24.31)$ in the confirmed group.

Table 1. Comparison of general information and clinical symptoms between the two groups.

\begin{tabular}{cccccc}
\hline & All patients & Suspected group & Confirmed group & & \\
Clinical features & $(\mathrm{n}=168)$ & $(\mathrm{n}=81)$ & $(\mathrm{n}=87)$ & & P value \\
\hline Male (\%) & $93(55.36)$ & $44(54.32)$ & $49(56.32)$ & 0.068 & 0.877 \\
\hline Age (years) & $39(31-54)$ & $36(29-50)$ & $45(35-56)$ & 2.496 & 0.013 \\
\hline Clinical symptoms & & & & & \\
\hline Fever (\%) & $129(76.79)$ & $58(71.61)$ & $71(81.61)$ & 2.355 & 0.145 \\
Cough (\%) & $108(64.29)$ & $57(70.37)$ & $51(58.62)$ & 2.522 & 0.147 \\
Fatigue (\%) & $33(19.64)$ & $12(14.82)$ & $21(24.14)$ & 2.310 & 0.173 \\
Sore throat (\%) & $27(16.07)$ & $15(18.52)$ & $11(12.64)$ & 1.107 & 0.394 \\
Runny nose congestion (\%) & $17(10.12)$ & $8(9.88)$ & $9(10.35)$ & 0.010 & 1.000 \\
Muscle aches (\%) & $22(13.10)$ & $4(4.94)$ & $18(20.69)$ & 9.145 & 0.003 \\
Anorexia and diarrhea (\%) & $20(11.91)$ & $6(7.41)$ & $14(16.09)$ & 3.017 & 0.098 \\
Headache and dizziness (\%) & $19(11.31)$ & $11(13.58)$ & $8(9.20)$ & 0.804 & 0.467 \\
Chest tightness (\%) & $17(10.12)$ & $5(6.17)$ & $12(13.79)$ & 2.678 & 0.127 \\
Chest pain (\%) & $7(4.17)$ & $6(7.41)$ & $1(1.15)$ & 4.114 & 0.057 \\
\hline
\end{tabular}


In chest $\mathrm{CT}$ imaging omics, ground-glass shadows and lesions infiltrating extrapulmonary bands were the most common in the two groups of patients. The suspected group was $36(44.44 \%)$ and $42(51.85 \%)$, which was less than 64 (73.56\%) in the confirmed group.) and $82(94.25 \%)$, the difference is significant $(P<0.001)$. In addition, the median number of bronchial vascular bundle thickening, interlobular thickening, and disease infiltrating lung lobes in the suspected group were less than those in the confirmed group, and the former were $6(7.41 \%), 5(6.17 \%)$, and $2(1,3)$, the latter are $50(57.47 \%), 33(37.93 \%)$ and $3(2,4)$, the difference is statistically significant $(P<0.001)$. The median of small nodules in the suspected group was $15(18.52 \%)$, and the median of small nodules in the confirmed group was $34(39.08 \%)$, the difference was significant $(P=0.003)$. However, there was no significant difference in pleural effusion and air bronchial signs between the two groups. See Table 2 for details.

\subsection{Factors Related to the First Diagnosis of Clinical Manifestations of Ordinary 2019-nCoV}

Taking the 2019-nCoV as the dependent variable and the possible influencing factors in clinical manifestations as the independent variables, the unconditional binary logistic regression was used to perform multivariate regression analysis. The independent variable elimination level was 0.05 , and there were missing data in the first diagnosis. PCT has also been eliminated, and the hazard ratio (HR) and its $95 \% \mathrm{CI}$ estimate the intensity of the influence of related factors.

The regression analysis results (see Table 3) showed that the general data of the patient, fever and muscle aches in the clinical symptoms, ground glass shadow, bronchial blood vessel thickening, and lesion infiltration outside the lung are common 2019-nCoV in the imaging omics. Independent risk factors; WBC count in blood routine, cough, sore throat and headache in clinical symptoms are independent negatively correlated factors of common 2019-nCoV.

\subsection{Establish LOGISTIC Regression Equation}

Perform binary logistic unconditional regression on clinical data, and get the probability equation for predicting 2019-nCoV: $P=\mathrm{e}^{x} /\left(1+\mathrm{e}^{x}, x=-6.226+0.071\right.$ $\times$ age (years) $+1.72 \times$ Fever $+2.858 \times$ muscle soreness $+2.131 \times$ ground glass shadow $+3.000 \times$ bronchial vascular bundle thickening $+3.438 \times$ lesion infiltration outside the lungs- $0.304 \times$ blood routine WBC count- $1.478 \times$ cough $-1.83 \times$ sore throat- $2.413 \times$ headache.

Among them, e is the logarithm of the natural number, the age unit is one year old, the white blood cell count is derived from the peripheral blood routine $\left(\times 10^{9} / \mathrm{L}\right)$, fever, muscle aches, cough, sore throat, headache, ground glass shadow, bronchial vascular bundle Thickening and lesions infiltrating the lung extracorporeal zone were calculated by two classifications ( $0=$ none, $1=$ yes).

Calculate the predictive value of ordinary $2019-\mathrm{nCoV}$ for the above-mentioned 168 patients, and draw the ROC curve with nucleic acid test results as the gold standard (Figure 1). The area under the ROC curve (AUC) is calculated to be 
0.945 (0.915 - 0.976). Choosing an appropriate critical value of 0.360 , we get sensitivity $=0.920$ and specificity $=0.827$.

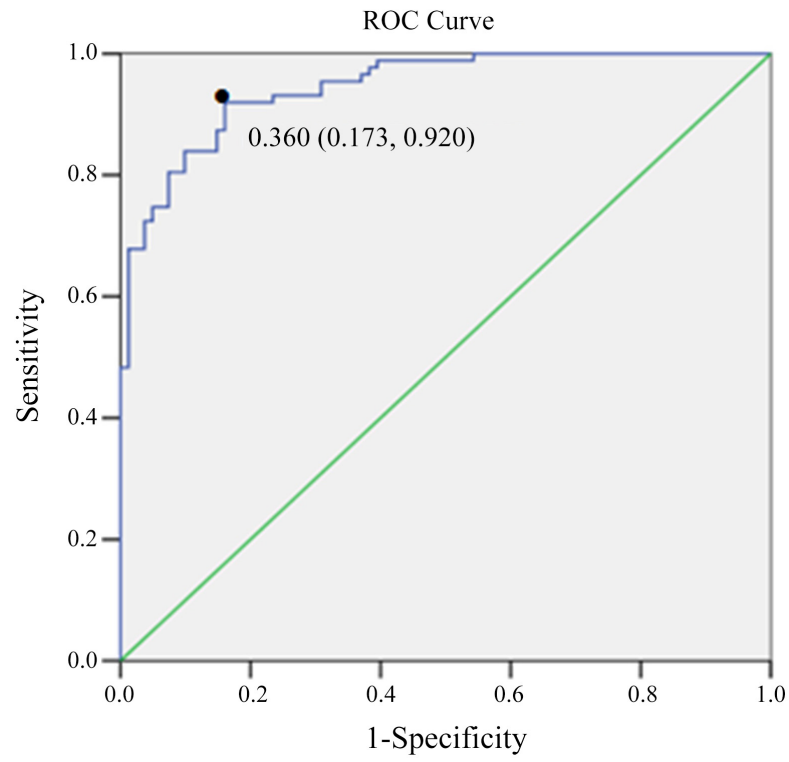

Figure 1. The ROC curve of the first diagnosis prediction model for the clinical manifestations of ordinary 2019-nCoV.

Table 2. Comparison of auxiliary examinations between the two groups.

\begin{tabular}{|c|c|c|c|c|}
\hline \multirow{2}{*}{ Check index } & Suspected group & Confirmed group & \multirow{2}{*}{$\mathrm{Z} / \mathrm{X}^{2}$} & \multirow{2}{*}{$P$ value } \\
\hline & $(\mathrm{n}=81)$ & $(\mathrm{n}=87)$ & & \\
\hline WBC $\left(\times 10^{9} / \mathrm{L}\right)$ & $7.00(4.79,8.55)$ & $5.40(4.20,6.73)$ & 3.965 & $<0.001$ \\
\hline NEUT $\left(\times 10^{9 /} \mathrm{L}\right)$ & $4.85(3.17,6.49)$ & $3.40(2.43,4.49)$ & 3.746 & $<0.001$ \\
\hline LYM $\left(\times 10^{9} / \mathrm{L}\right)$ & $1.17(0.87,1.63)$ & $1.19(0.93,1.54)$ & 0.192 & 0.848 \\
\hline CRP (mg/l) & $18.35(6.60,43.80)$ & $9.81(4.43,24.31)$ & 1.895 & 0.058 \\
\hline PCT (ng/ml) & $0.26(0.14,0.35)$ & $0.12(0.05,0.26)$ & 2.539 & 0.011 \\
\hline \multicolumn{5}{|l|}{ Chest CT imaging omics } \\
\hline Ground glass shadow (\%) & $36(44.44 \%)$ & $64(73.56 \%)$ & 14.762 & $<0.001$ \\
\hline $\begin{array}{c}\text { Lesion infiltrates } \\
\text { the outer lung area (\%) }\end{array}$ & $41(50.62 \%)$ & $82(94.25 \%)$ & 40.777 & $<0.001$ \\
\hline $\begin{array}{c}\text { Bronchial vascular } \\
\text { bundle thickening (\%) }\end{array}$ & $6(7.41 \%)$ & $50(57.47 \%)$ & 47.310 & $<0.001$ \\
\hline Interlobular thickening (\%) & $5(6.17 \%)$ & $33(37.93 \%)$ & 24.171 & $<0.001$ \\
\hline $\begin{array}{c}\text { Number of infiltrating } \\
\text { lung lobes }\end{array}$ & $2(1,3)$ & $3(2,4)$ & 4.449 & $<0.001$ \\
\hline Small nodules (\%) & $15(18.52 \%)$ & $34(39.08 \%)$ & 8.584 & 0.004 \\
\hline Combined pleural effusion (\%) & $5(6.17 \%)$ & $3(3.45 \%)$ & 0.687 & 0.484 \\
\hline Air bronchial sign (\%) & $9(11.11 \%)$ & $5(5.75 \%)$ & 1.580 & 0.268 \\
\hline
\end{tabular}


Table 3. Multivariate logistic regression analysis of clinical manifestations of common 2019-nCoV.

\begin{tabular}{ccccccc}
\hline factor & $\begin{array}{c}\text { Regression } \\
\text { coefficients }\end{array}$ & $\begin{array}{c}\text { Standard } \\
\text { confidence }\end{array}$ & Forest & $P$ & Exp (B) & $\begin{array}{c}95 \% \\
\text { Interval CI }\end{array}$ \\
\hline age & 0.071 & 0.020 & 12.197 & $<0.001$ & 1.073 & $1.032-1.117$ \\
WBC $\left(\times 10^{9 /} \mathrm{L}\right)$ & -0.304 & 0.097 & 9.728 & 0.002 & 0.738 & $0.610-0.893$ \\
cough & -1.478 & 0.634 & 5.438 & 0.020 & 0.228 & $0.066-0.790$ \\
Sore throat & -1.830 & 0.875 & 4.371 & 0.037 & 0.160 & $0.029-0.890$ \\
fever & 1.720 & 0.701 & 6.013 & 0.014 & 5.584 & $1.412-22.077$ \\
headache & -2.413 & 1.038 & 5.407 & 0.020 & 0.090 & $0.012-0.684$ \\
$\begin{array}{c}\text { Muscle ache } \\
\text { Ground glass shadow }\end{array}$ & 2.858 & 1.097 & 6.784 & 0.009 & 17.434 & $2.029-149.822$ \\
$\begin{array}{c}\text { Bronchial vascular } \\
\text { bundle thickening }\end{array}$ & 3.000 & 0.707 & 18.016 & $<0.001$ & 20.082 & $5.026-80.240$ \\
$\begin{array}{c}\text { Lesion infiltrates the } \\
\text { outer lung }\end{array}$ & 3.438 & 0.849 & 16.381 & $<0.001$ & 31.122 & $5.889-164.475$ \\
Constant & -6.226 & 1.560 & 15.931 & $<0.001$ & 0.002 & \\
\hline
\end{tabular}

\section{Discussion}

This study compared the general data of 168 patients with common suspected cases of $2019-\mathrm{nCoV}$ and confirmed cases, and found that the median age of common confirmed cases of 2019-nCoV was 45 (35 - 56) years old, which is consistent with literature reports [6], and more than the normal suspected case of 2019-nCoV.

In this study, the clinical symptoms were more common with fever and cough, but there was only a significant difference in muscle soreness between the confirmed group and the suspected group $(p=0.003)$. The number of confirmed cases of ordinary 2019-nCoV reached 18 (20.69\%), the suspected cases have only $4(4.94 \%)$, and there were no significant differences in other symptoms. Xiong Xiaoming reported that the concentration of serum lactate dehydrogenase in confirmed 2019-nCoV cases is higher than that in cases excluded from 2019-nCoV [4]. NAD-dependent lactate dehydrogenase can promote the production of lactic acid, and a certain concentration of lactic acid in the muscle space may cause the feeling of muscle aches. Of course, there is a certain degree of uncertainty in the symptoms complained by patients. The results of this study suggest that clinicians can fully consider "febrile, dry cough, fatigue and other symptoms" in accordance with the National Health Commission's series of 2019-nCoV diagnosis and treatment guidelines, so that there is almost no difference in clinical symptoms between suspected cases and confirmed cases, which are relatively rare. The symptoms of "muscle soreness" deserve attention.

This study also compared the commonly used laboratory examination data of 168 patients between common suspected cases and confirmed cases, and found 
that the median white blood cell count (WBC, $\times 10^{9} / \mathrm{L}$ ), medium sex granulocyte count (NEUT, $\times 10^{9} / \mathrm{L}$ ), lymphocyte count $\left(\mathrm{LYM}, \times 10^{9} / \mathrm{L}\right)$ and PCT $(\mathrm{ng} / \mathrm{ml})$ are all within the normal range, but the median white blood cell count, neutrophil count, and PCT levels in the peripheral blood of the confirmed case were 5.40 $(4.20,6.73), 3.40(2.43,4.49)$, and $0.12(0.05,0.26)$ respectively, which were all lower than the $7.00(4.79,8.55), 4.85(3.17,6.49)$ and $0.26(0.14,0.35)$ of suspected cases (the difference was significant $(P<0.001, P<0.001, P=0.011)$ ). The median lymphocyte count (LYM, $\left.\times 10^{9} / \mathrm{L}\right)$ and CRP $(\mathrm{mg} / \mathrm{l})$ of the two groups were not significantly different. It has been reported in the literature [7] [8] that in patients with severe 2019-nCoV, peripheral blood lymphocyte counts are lower, and CRP and PCT levels are higher. The confirmed cases and suspected cases of 2019-nCoV in this study are all common types, and the peripheral blood white blood cell count, neutrophil count, lymphocyte count and PCT are all within the normal range, indicating that the body's inflammatory response in all cases is relatively not serious. At the same time, the results of this study show that the peripheral blood median white blood cell count, neutrophil count, and PCT levels of suspected 2019-nCoV cases are higher than those of confirmed cases, suggesting that more cases in the suspected group have bacterial infections. The Committee's series of 2019-nCoV diagnosis and treatment guidelines may not completely exclude bacterial pneumonia in clinical practice.

In this study, after comparing the chest CT imaging of 168 patients with COVID-19 common suspected cases and confirmed cases, it was found that only pleural effusion and air bronchial signs were not significantly different between the two groups. The number of lesions infiltrating the outer pulmonary band, the thickening of the bronchial vascular bundle, the thickening of the interlobular septum, the number of infiltrating lung lobes and the shadow of small nodules were all higher than those in the suspected group, and the difference was significant $((P<0.001$ for the first 5 items, $P<0.001$ for small nodules $)=0.004)$, the former are 64 (73.56\%), 82 (94.25\%), 50 (57.47\%), 33 (37.93\%), $3(2,4)$ and $34(39.08 \%)$, the latter are $36(44.44 \%), 41$ (50.62\%), 6 (7.41\%), 5 (6.17\%), 2 (1, $3)$ and $15(18.52 \%)$. It has been reported in the literature that ground-glass density shadows, lesions along the bronchial vascular bundles, and thickening of the internal lobules help to distinguish between 2019-nCoV patients and suspected patients [9] [10]; due to the rich blood flow in the subpleural pulmonary lobules, the virus is easier Invasion, the GGO of patients with $2019-\mathrm{nCoV}$ is mostly distributed along the subpleural [10]; the first diagnosis of 2019-nCoV lung CT is characterized by ground glass and nodular shadows located under the pleura [11]; The number of lesions of chest CT of pneumonia infected by $2019-\mathrm{nCoV}$ is higher than other pneumonia infections [12]. Obviously, the chest CT imaging omics results of this study are consistent with the above literature reports.

After fully introducing various factors of clinical manifestations and performing binary logistic unconditional regression analysis, this study established a general 2019-nCoV first diagnosis prediction mathematical model, and the area under the ROC curve (AUC) reached 0.945 (0.915 - 0.976). With the best 
critical value of $36 \%$, the sensitivity of the prediction model is 0.920 and the specificity is 0.827 . The accuracy of the diagnosis is good, and it has good predictive significance.

This study is a retrospective case-control study. The clinical data are enrolled in their first diagnosis. The number and source of samples have specific regional limitations. These limitations need to be improved and supplemented in future studies.

\section{Conflicts of Interest}

The authors declare no conflicts of interest regarding the publication of this paper.

\section{References}

[1] Miller, T.E., Garcia Beltran, W.F., Bard, A.Z., Gogakos, T., Anahtar, M.N., Astudillo, M.G., Yang, D., Thierauf, J., Fisch, A.S., Mahowald, G.K., Fitzpatrick, M.J., Nardi, V., Feldman, J., Hauser, B.M., Caradonna, T.M., Marble, H.D., Ritterhouse, L.L., Turbett, S.E., Batten, J., Georgantas, N.Z., Alter, G., Schmidt, A.G., Harris, J.B., Gelfand, J.A., Poznansky, M.C., Bernstein, B.E., Louis, D.N., Dighe, A., Charles, R.C., Ryan, E.T., Branda, J.A., Pierce, V.M., Murali, M.R., Iafrate, A.J., Rosenberg, E.S. and Lennerz, J.K. (2020) Clinical Sensitivity and Interpretation of PCR and Serological COVID-19 Diagnostics for Patients Presenting to the Hospital. FASEB Journal, 34, 13877-13884. https://doi.org/10.1096/fj.202001700RR

[2] Zheng, Z., et al. (2020) The Diagnosis of SARS-CoV2 Pneumonia: A Review of Laboratory and Radiological Testing Results. Journal of Medical Virology, 92, 2420-2428. https://doi.org/10.1002/jmv.26081

[3] National Health Council (2020) New Coronavirus Pneumonia Diagnosis and Treatment Plan (Trial Version 7). http://www.nhc.gov.cn/yzygj/s7653p/202003/46c9294a7dfe4cef80dc7f5912eb1989.s html?wynorhhjir4y=1591457765637

[4] Xiong, X., Ouyang, M., Zhang, H., Wang, Y., Xia, X., Wang, Y., Liao, B., Feng, X., Tong, G., Liu, X., Shi, D. and Yang, C. (2020) Analysis of the Early Clinical Characteristics of COVID-19 and N-COVID-19. Practical Preventive Medicine, 36, 991-995.

[5] Chu, D.K.W., et al. (2020) Molecular Diagnosis of a Novel Coronavirus (2019nCoV) Causing an Outbreak of Pneumonia. Clinical Chemistry, 66, 549-555.

[6] Dai, Z., Gao, L., Luo, W., Xiao, J., Huang, C., Zeng, G., Liu, Z., Zhao, S., Yang, H., Sun, Q., Zhang, H., Wang, J. and Hu, S. (2020) Clinical Characteristics of Coronavirus Disease 2019 in Hunan Province. Practical Preventive Medicine, 27, 396-399. https://doi.org/10.1093/clinchem/hvaa029

[7] Wei, L., Gao, W., Su, X., Zheng, X., Yang, X., Liu, M. and Xu, B. (2020) Differential Analysis of Clinical Characteristics and Prognosis of Patients with Severe and Non-Severe Coronavirus Disease. Journal of PLA Medical College, 1-5. http://kns.cnki.net/kcms/detail/10.1117.R.20200720. 0910.004.html

[8] Tang, J., Xuan, C., Lin, J., Zhang, E., Zhong, M. and Deng, J. (2020) Clinical Significance of Detecting C-Reactive Protein, Interleukin-6 and Procalcitonin in COVID-19. The Journal of Practical Medicine, 36, 839-841.

[9] Chung, M., Bernheim, A., Mei, X., et al. (2020) CT Imaging Features of 2019 Novel Coronavirus (2019-nCoV). Radiology, 295, 202-207. 
https://doi.org/10.1148/radiol.2020200230

[10] Li, Y. and Xia, L.M. (2020) Coronavirus Disease 2019 (COVID-19): Role of Chest CT in Diagnosis and Management. American Journal of Roentgenology, 214, 1280-1286. https://doi.org/10.2214/AJR.20.22954

[11] Liu, H., Zhang, D., Yang, Y., Long, B., Yin, L., Zhao, M. and Peng, Y. (2020) Analysis of the Initial Chest High Resolution CT Manifestations of COVID-19. Chinese Journal of Radiology, 54, E007-E007.

[12] Zhang, Y., Nan, C., Liu, H., Li, H., Yang, H. and Liu, H. (2020) Re-Discussion about CT Signs of Novel Coronavirus Pneumonia. Clinical Focus, 35, 106-112. 